\title{
Temporal variation of noise correlation function (NCF) in Beijing and surroundings: Its relation with climate events and implications*
}

\author{
Changsheng Jiang ${ }^{1}$ and Zhongliang $\mathrm{Wu}^{1,2, \uparrow}$ \\ ${ }^{1}$ Institute of Geophysics, China Earthquake Administration, Beijing 100081, China \\ ${ }^{2}$ Laboratory of Computational Geodynamics, Graduate University of Chinese Academy of Sciences, Beijing 100049, China
}

\begin{abstract}
Noise correlation function (NCF) was calculated using the data of the Beijing Capital-Area Telemetered Digital Seismograph Network from June 12 to September 12, 2005. Signal-to-noise ratio (SNR) is used to characterize the quality of $\mathrm{NCF}$ at each station pair. The SNR (in $\mathrm{dB}$ ) is shown to be dependent on the separation distance $R$ of the station pair via $\mathrm{SNR}=$ $A-B \log R$. 'Normalized average SNR' for all the station pairs can then be calculated, as represented by the value of SNR taking $R=250 \mathrm{~km}$ in the empirical SNR- $R$ relation, to measure the overall quality of the NCF result. The 'normalized average SNR' of the NCF shows temporal variation and is apparently dependent on the root-mean-square (RMS) velocity of the microseism. The result obtained by this experiment provides clues to the explanation of the properties of NCF, such as the dominant mechanism underlying (diffuse wave fields or uncorrelated sources), and the dependence of SNR on the time length of recordings.
\end{abstract}

Key words: noise correlation function; Beijing and surroundings; signal-to-noise ratio CLC number: P315 Document code: A

\section{Introduction}

Since recent years, using noise correlation function (NCF) to retrieve Green's function (Weaver and Lobkis, 2001; Snieder, 2004; Wapenaar, 2004) has caused widespread attention in acoustics (e.g., Weaver and Lobkis, 2004; Roux et al, 2004, 2005; Wapenaar et al, 2005) and geophysics (e.g., Campillo and Paul, 2003; Shapiro and Campillo, 2004; Sabra et al, 2005a, b; Shapiro et al, 2005) due to its significant potential for application. It is shown that the cross-correlation of microseism recordings can be used to infer the impulse response between receivers, i.e., Green's function (Weaver and Lobkis, 2001; Snieder, 2004; Wapenaar, 2004), providing seismological exploration of the Earth's structure with an innovative tool without the using of earthquakes and/or explosions. Up to now, theoretical derivations imply several options of the underlying mechanism of the NCF approach, among which two main options are diffuse wave fields generated by

\footnotetext{
* Received 11 September 2007; accepted in revised form 25 November 2008 ; published 10 February 2009.

* Corresponding author. e-mail: wuzhl@gucas.ac.cn
}

multiple scattering, or multiple uncorrelated sources (Sanchez-Sesma et al, 2006). Identification of which mechanism may be predominant needs some designed experiments by real seismic data. Applying the NCF approach to Beijing, we tried to get some clues to the answer of this question by investigating the temporal variation of the quality of NCF, especially its relation with some known events such as Western North Pacific typhoons.

\section{Data used}

We used the data from the Beijing Capital-Area Telemetered Digital Seismograph Network, located in North China, deployed since the year 2000 (Guo et al, 2002), as shown in Figure 1. The network is near the Bohai Sea which acts as the main source of the microseism, very similar to the case of one-sided illumination as discussed by Wapenaar (2006). The network has 44 three-component broadband seismograph stations with flat velocity response from $0.05 \mathrm{~Hz}$ to $20 \mathrm{~Hz}$ and 5 three-component very-broadband seismograph stations with $120 \mathrm{~s}$ to $20 \mathrm{~Hz}$ flat ground velocity response, as shown in Figure 2a. The instruments are designed and 


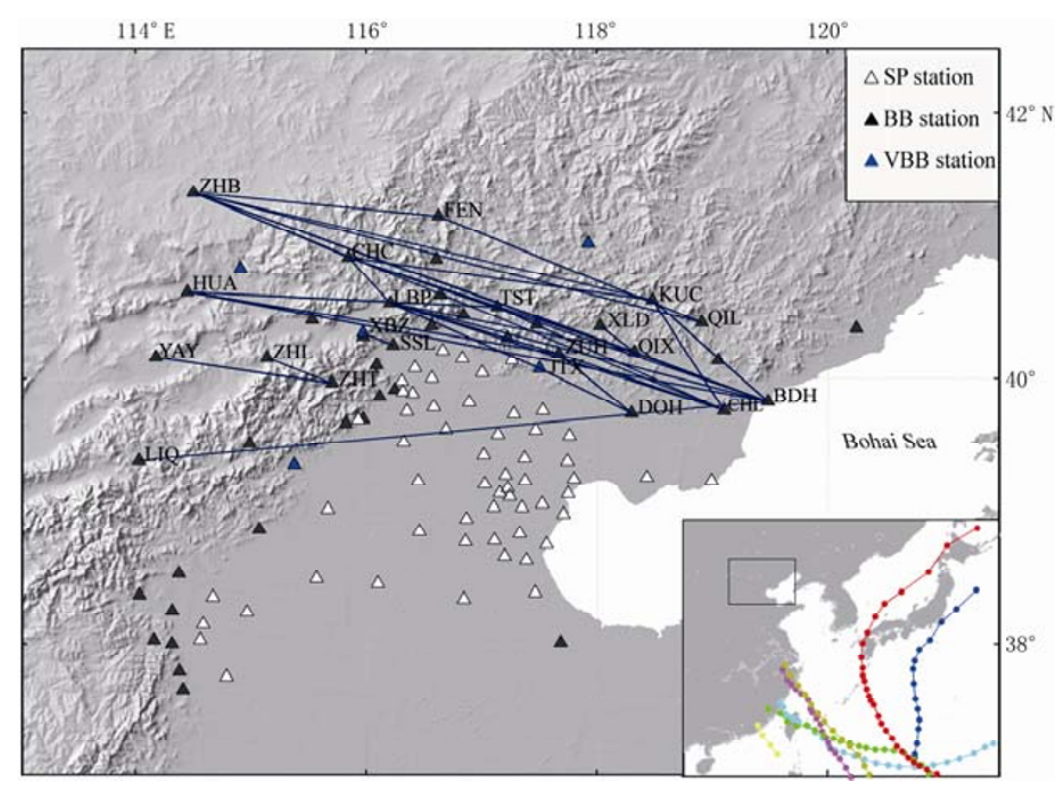

Figure 1 Distribution of seismic stations in Beijing Capital-Area Telemetered Digital Seismograph Network. Seismograms from $21 \mathrm{BB} / \mathrm{VBB}$ stations are used in the calculation, with their links shown in the figure by the blue lines. There were 32 station pairs, approximately perpendicular to the coastline, having good quality NCFs. Other station pairs failed to get acceptable NCFs. Index map shows the location of the studied area in north China and the center tracks of 7 Western North Pacific typhoons from June 12 to September 12, 2005.
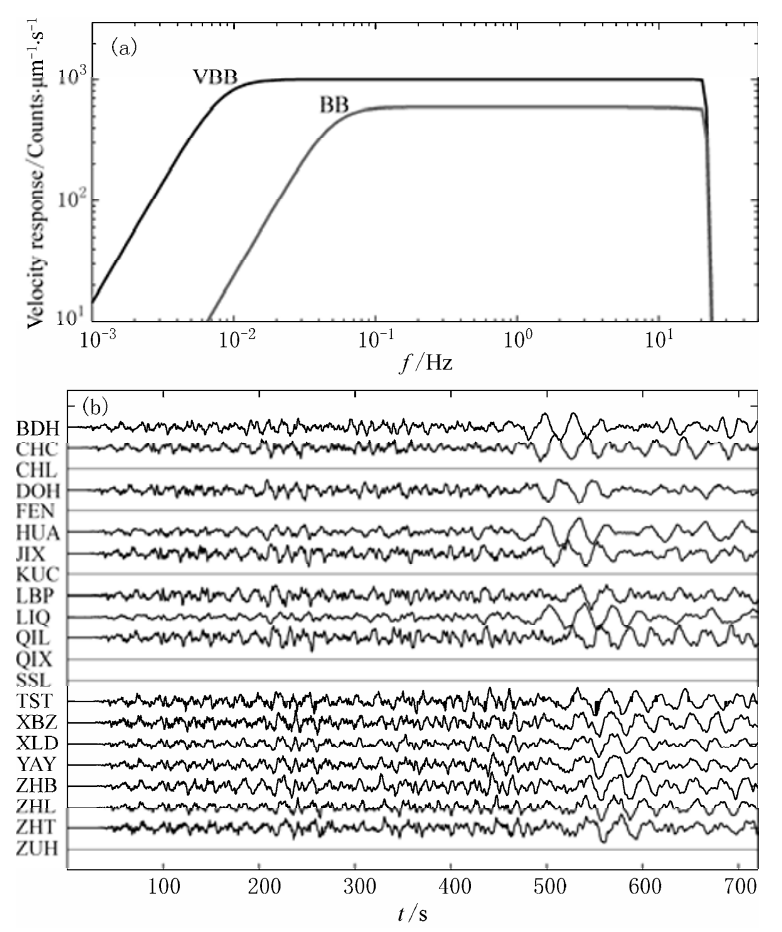

Figure 2 Characteristics of the instruments. (a) Frequency response curve of the broadband $(\mathrm{BB})$ and very broadband (VBB) seismographs used in the Beijing Capital-Area Telemetered Digital Seismograph Network; (b) Waveforms of the December 26, 2004, off the west coast of northern Sumatra, Indonesia, $M_{\mathrm{W}} 9.0$ earthquake recorded by 19 broadband stations and 2 very broadband stations (XBZ and JIX), showing the characteristics and performance of the network. The seismograms are aligned according to the order of the epicentral distance. produced by Beijing GeoDevice Co. (Gang-Zhen Company). Among all these stations, there are $21 \mathrm{BB} / \mathrm{VBB}$ stations available to calculate the NCF with ideal configuration of station pairs and good quality of data continuity, as shown in Figure 1. As a demonstration of the characteristics and performance of the network, Figure $2 \mathrm{~b}$ shows the vertical component waveforms of the December 26, 2004, off the west coast of northern Sumatra, Indonesia, $M_{\mathrm{W}} 9.0$ earthquake, recorded by the 19 broadband stations and 2 very brriod, from June 12 to September 12, 2005, to conduct the experiment. According to 'CMA-STI Best Track Dataset for Tropical Cyclones in the Western North Pacific' obtained from Shanghai Typhoon Institute of the China Meteorological Administration (http://www.typhoon.gov.cn), there are seven Western North Pacific typhoons occurred during this period, providing a very good set of 'known events'. The index map of Figure 1 shows their center tracks, respectively.

\section{Analysis method}

Processing of digital seismograms to retrieve NCF has been systematically discussed by Bensen et al (2007). Here we follow the calculation procedure of NCF as proposed by Sabra et al $(2005 \mathrm{a}, \mathrm{b})$ in which the cross correlation coefficient $C_{i j}$ is computed from the continuous seismograms $v_{i}\left(r_{1}, t\right)$ and $v_{j}\left(r_{2}, t\right)$ of micro- 
seism by integrating over the whole time duration $T$ :

$$
C_{i j}(1,2, t)=\int_{0}^{T} v_{i}\left(r_{1}, \tau\right) v_{j}\left(r_{2}, \tau+t\right) \mathrm{d} \tau,
$$

where $v_{i}\left(r_{1}, t\right)$ and $v_{j}\left(r_{2}, t\right)$ are the continuously recorded broadband seismic data at stations 1 (located at $r_{1}$ recording component $i$ ) and 2 (located at $r_{2}$ recording component $j$ ), respectively, in the days without earthquakes recorded, and $T$ is the total cross-correlation time, being taken as 24 hours in this study. Sabra et al (2005a) has shown by real data that SNR increases proportional to the square root of recording time. Therefore taking 24 hours does not loss generality for discussing the quality of NCF in this study. In our analysis vertical component seismograms are used. Preprocessing of the data includes three steps: re-sampling of the data with a $10 \mathrm{sps}$ sampling rate; filtering with a $0.05-0.2 \mathrm{~Hz}(5-20 \mathrm{~s})$ band-pass filter; and taking of the one-bit signal to avoid the over-emphasis of large amplitude noise, as described in detail by Campillo and Paul (2003) and Larose et al (2004).

\section{4 'Normalized average SNR' measure of the SNR of NCF}

Figure 3 shows an example of the NCF estimation. It can be seen that the quality of NCF varies with time. In quantifying the quality of the NCF, we use the SNR, which is defined as the ratio of the maximum amplitude of the main surface-wave-like arrival and the root-mean-squares value within an incoherent noise-only time window. Here the 'main surface-wave-like arrival' is chosen as the seismic phase with apparent velocity $2.6-3.6 \mathrm{~km} / \mathrm{s}$, as indicated by the shadow zone in Figure 3a; The 'incoherent noise-only time window' is selected, somehow arbitrarily but without losing of generality, as the time period from $-200 \mathrm{~s}$ to $-150 \mathrm{~s}$ in Figure 3a. In Figure 3 all the traces use the same scale; therefore weak NCFs cannot be visible as a result of the comparison with the 'best' NCF, although most of them still have the SNR larger than 6 $\mathrm{dB}$ as indicated by Figure $3 \mathrm{~b}$.

Figure 4 gives an example of the empirical relation between the SNR and the distance $R$ of the station pair separation. Although the data points are scattered in the diagram, an empirical relation can still be obtained, as represented by

$$
\mathrm{SNR}=A-B \log R,
$$

in which $A$ and $B$ are constants. In the fitting in Figure 4, we get $A \approx 37$ and $B \approx 3.5$ if SNR is in $\mathrm{dB}$ and $R$ is in $\mathrm{km}$. Note that equation (2) is not very much interesting in physics because, considering the definition of SNR, this relation means that the dependence of the ratio between signal and noise amplitude on the separation distance is weak, say $R^{-0.2}$. The significance of equation (2) is to provide a 'normalized SNR' so that station pairs with different distances of separation could be compared with each other. Moreover, a 'normalized average value' of SNR for all the station pairs can be calculated by firstly fitting the SNR- $R$ relation via equation (2) and then taking $R$ as the 'standard value' $250 \mathrm{~km}$. Value $250 \mathrm{~km}$ is somehow arbitrarily taken, only for the comparison of different station pairs with different separation distances. All these considerations, seemingly not as straightforward as one expected, are only from a simple reason that by definition SNRs cannot be added with each other, therefore simply taking the 'average' of SNR is problematic.

\section{Temporal variation of the 'normal- ized average $S N R$ '}

Figure 5 shows the relation between the "normalized average value' of SNR at each day and the average level of microseism at the same day. Figure 5a shows the root-mean-square (RMS) ground velocity of the ambient noise, or microseism, for each day from June 12 to September 12, 2005, for all the stations, as indicated by the gray dots. The average of all the stations for each day is also calculated, as indicated by the heavy solid line. By its nature, the variation of the level of noise/microseism is determined by climate and oceanic factors such as temperature and pressure of the atmosphere and the weather in the sea area. Figure $5 \mathrm{~b}$ shows, for each station pair, the SNR value as gray dots. The 'normalized average value' of SNR for all the stations at each day is shown by the heavy solid line. From the comparison between Figures 5a and 5b, a logarithmic correlation between the RMS velocity of microseism and the 'normalized average SNR' of the NCF can be observed. Figure 6 shows such a correlation. From the figure it may be seen that the relation can be divided into two parts. For low-SNR NCF, there is not clear correlation between the RMS velocity of microseism and the 'normalized average SNR' of the NCF. For the NCF with high-SNR (larger than $12 \mathrm{~dB}$ and above the gray dotted line), however, such a correlation is clear, as shown by the thick line. The investigation of factors 


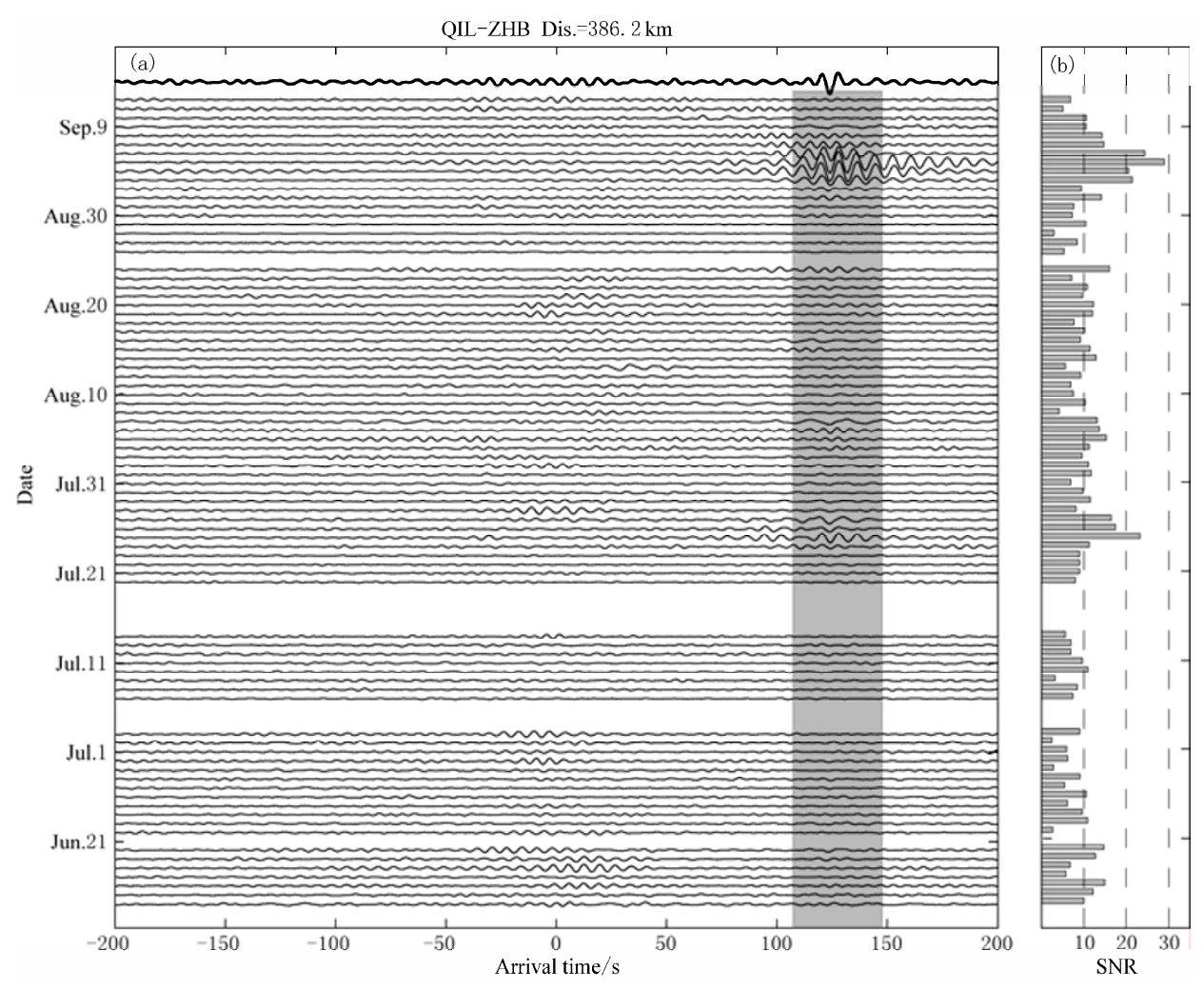

Figure 3 NCFs computed between stations QIL and ZHB (see Figure 2) using the continuous vertical channel recorded from June 12 to September 12, 2005. (a) NCF for every day from June 12 to September 12, 2005. Blank indicates lack of high-quality recordings or the day with earthquakes/explosions recorded. Top trace (thick line) shows the stacking of NCFs for this three-month time period. (b) Signal-to-noise ratio of the NCF varying with time. The SNR is defined as the ratio of the maximum amplitude of the surface-wave-like arrival, i.e., the seismic phase with apparent velocity $2.6-3.6 \mathrm{~km} / \mathrm{s}$ as indicated by the shadow zone in Figure $3 \mathrm{a}$, and the root-mean-squares for an incoherent noise-only time window, selected as the time period from $-200 \mathrm{~s}$ to $-150 \mathrm{~s}$ in Figure 3a. The unit of SNR is dB.

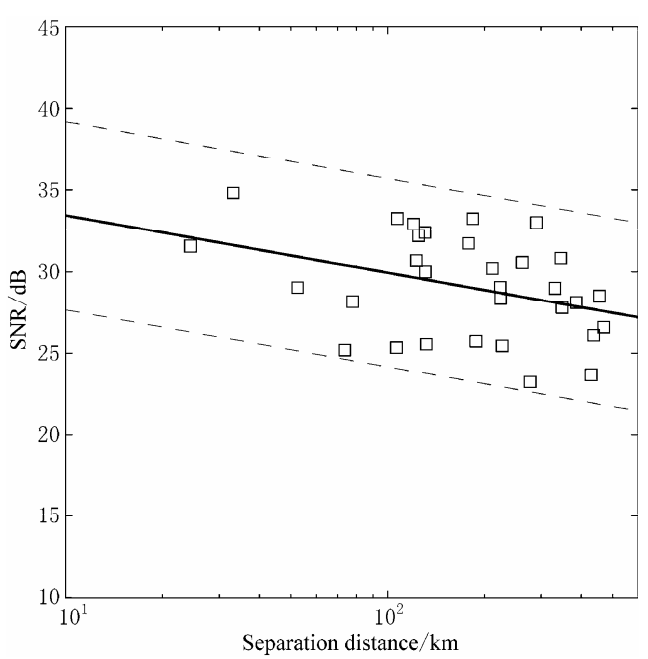

Figure 4 SNR of NCF versus the distance of station pair separation. The figure is for the stacked data from September 3 to 7,2005. Solid line indicates the fitting of the data points with a relation as shown in equation (2), and dashed lines shows the residual standard deviation around the fitted line. effecting the temporal variation of the microseism helps to understand the nature and variability of the ambient noise and the mechanism of NCF. Ritzwoller et al (2005) indicated that the microseisms occurring in shallow waters maybe results from the frequency doubling effect of the nonlinear wave-wave interaction between the incoming and reflected surface gravity waves. According to this, one possible clue is the typhoon impacts on Bohai Sea with average depth $25 \mathrm{~m}$. Figure 5a shows their duration time, indicating that microseism has a strong correlation with the typhoon strike.

\section{Conclusions}

Retrieving of structures using microseisms has been one of the important topics under discussion since recent years. In this paper we conducted an experiment studying the variation of the quality of NCF and its relation with climate events. Practically, the result obtained here is 

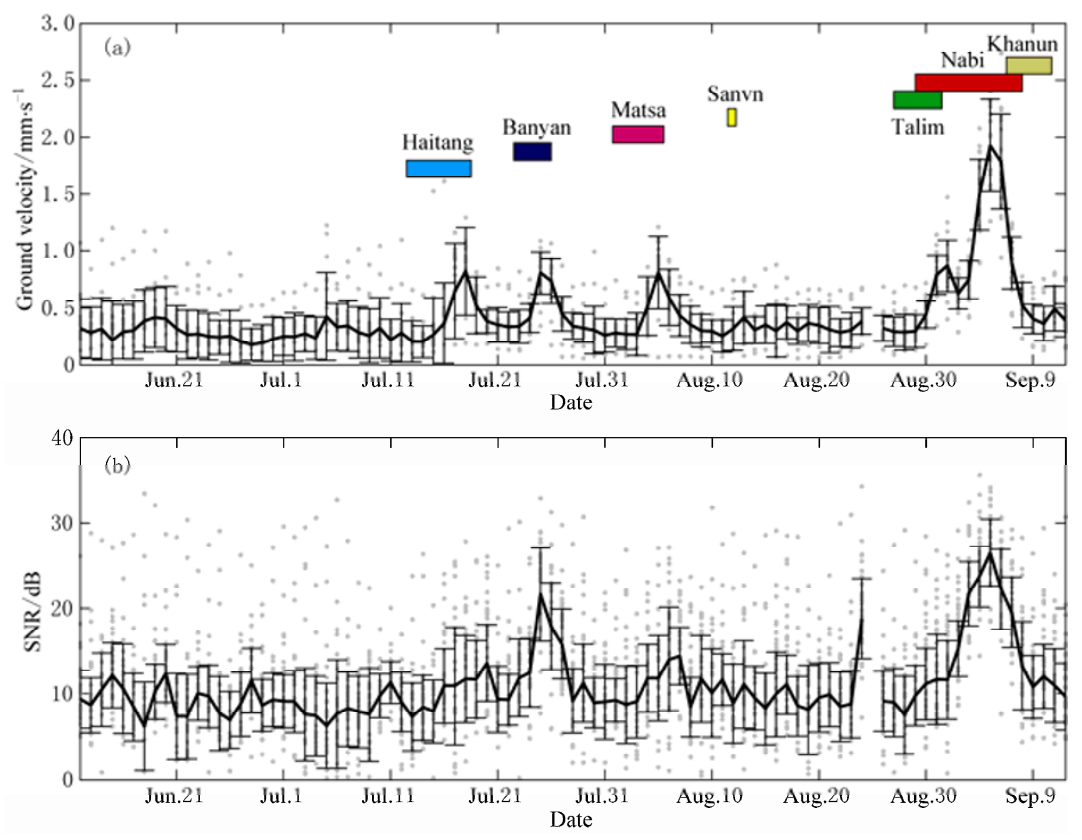

Figure 5 SNR versus level of microseism. (a) RMS ground velocity of ambient noise/microseism for each day and for all the stations, as indicated by each gray dot. Effects of earthquakes are eliminated before the calculation of the average ground velocity. Heavy solid line shows the average of all the stations for each day. The color blocks show the duration time window of Western North Pacific typhoons whose tracks are plotted in the index map of Figure 1; (b) SNR of NCF for each station pair, as represented by gray dots. The "normalized average value' of SNR is shown by the heavy solid line. See text for details.

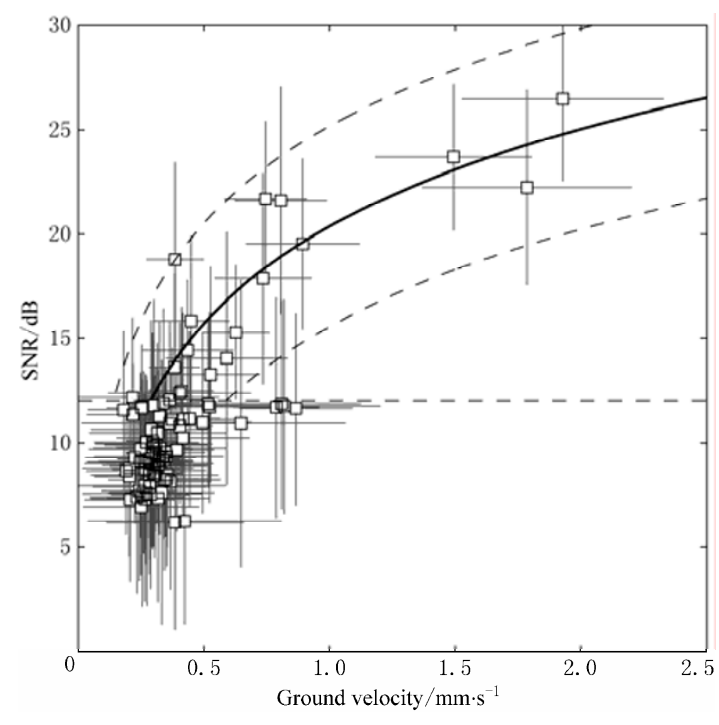

Figure 6 RMS velocity of microseism versus the "normalized average SNR' of the NCF. Solid line shows the relation between the RMS ground velocity and the SNR of the NCF for high-SNR NCF (with $\mathrm{SNR} \geq 12 \mathrm{~dB}$ ). See text for details. Black dashed lines show the residual standard deviation around the fitted line.

useful for the regions in which the SNR of NCF varies with time, such like the case of Beijing and its surround- ings: people can use the data within some specific time periods to obtain a high-quality NCF estimate. That is, based on the temporal variation of the 'illumination' of NCF as measured by the 'normalized average SNR', the 'brightest days' can be selected to obtain high-quality NCF results. Here the word 'illumination' comes from, e.g., Wapenaar (2006), who used the wordings of 'one-sided (and multi-sided) illumination' to show the source of the microseism. In our experiment, within the three months under study, there are five days with high 'illumination' of NCF. Figure 7 shows the NCF for all the station pairs under discussion, stacked for the 'brightest five days' from September 3 to 7, 2005. This week is notable in East Asia as the area was attacked by typhoon NABI, the largest typhoon in 2005. From the figure the surface-wave-like phases are clearly visible and traceable. Figure $7 \mathrm{~b}$ uses Hilbert transform to obtain the envelope of the seismograms. By fitting the relation between the arrival time of the peak of the envelope and the separation distance between the station pairs, apparent propagating velocity of the surface-wave-like phase can be estimated. Estimation of this apparent velocity obtains approximately $3.3 \mathrm{~km} / \mathrm{s}$, in accordance with the velocity of Rayleigh waves in this region. 

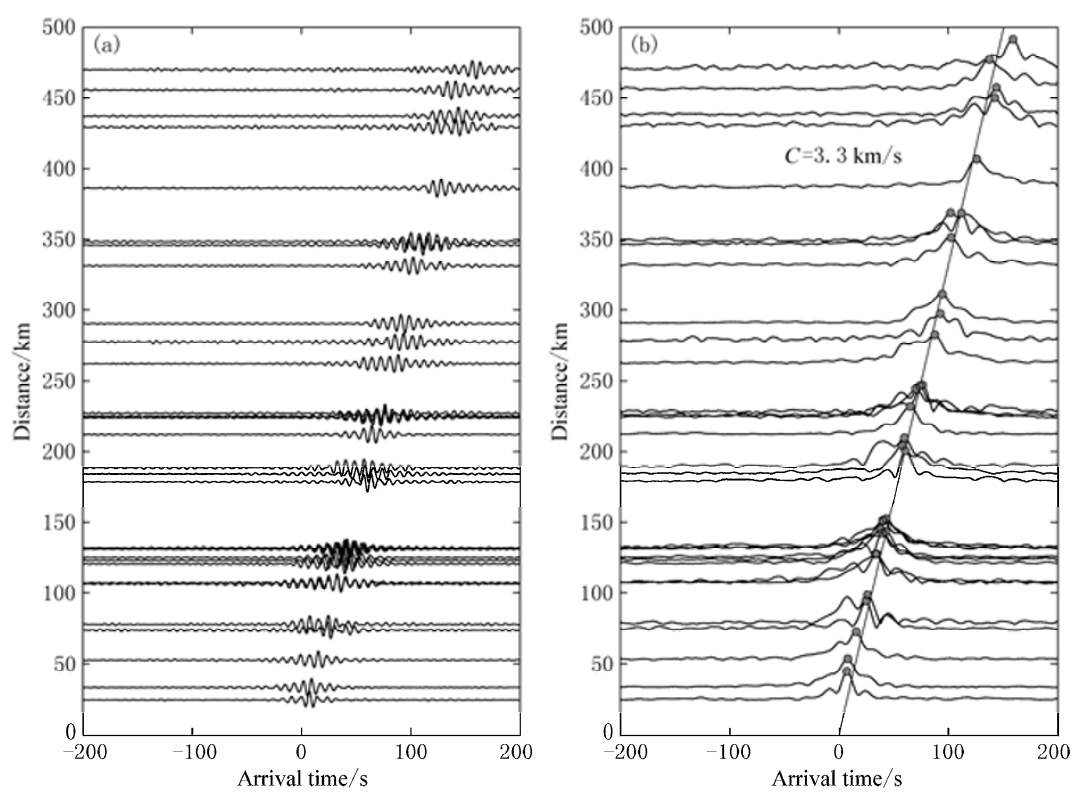

Figure 7 Cross section of NCFs changing with the separation distance of station pairs from 20 to $500 \mathrm{~km}$. (a) NCFs for different station pairs, stacked using the data within the 'brightest days' from September 3 to 7, 2005. Each trace has been normalized to the same amplitude; (b) Envelope of the NCF computed using Hilbert transform, in which peak values are shown by solid dots. Estimation of the apparent velocity $C$ by fitting the solid dots (as indicated by the straight line) gives a value of approximately $3.3 \mathrm{~km} / \mathrm{s}$, indicating that the NCFs are corresponding to Rayleigh waves.

\section{Discussion on the theoretical implica- tions}

At present, theoretical studies have provided several options of the underlying mechanism of the NCF approach. For example, two competing mechanisms are the diffuse wave fields generated by multiple scattering and the multiple uncorrelated sources (Sanchez-Sesma et al, 2006). From the temporal variation of the SNR of $\mathrm{NCF}$ and its dependence on the level of microseism, it seems that the multiple uncorrelated source hypotheses may be more straightforward. If the multiple scattering mechanisms dominate, then the dependence of SNR and the noise level will be weak, since the source and the scattering increase or decrease at the same time. On the other hand, if the uncorrelated source mechanism dominates, then the change of the situation of the source will affect the SNR significantly. The experiment of this paper shows the later case. Moreover, the dependence of SNR on the noise velocity level indicates the far-field nature of the signal and noise. This study is different from that of Tanimoto et al (2006) and Gerstoft and Tanimoto (2007) who studied the temporal variation of NCF. Rather, the focus of this study is not the temporal variation of NCF itself, but the temporal variation of its quality.

Another interesting aspect is the empirical dependence of total SNR on the time length of observation. Sabra et al (2005a, see their Figure 4) has shown by real data that SNR increases proportional to the square root of recording time. Although the methods for SNR calculation are different in details, qualitatively it can be understood why such relation holds. Due to the stochastic nature of seismic noise, its average over time is proportional to the square root of time length. The SNR of $\mathrm{NCF}$, as shown in Figure 6, is proportional to the average level of noise/microseism if asymptotic approximation is taken for the range between $15 \mathrm{~dB}$ and $25 \mathrm{~dB}$. In this case, SNR is proportional to the square root of time length. Our study also predicts that this relation holds only for high-SNR NCFs.

Acknowledgements This study is supported by the Fundamental Research and Development of Institute of Geophysics, China Earthquake Administration (DQJB07B03). Thanks are due to the Beijing Capital-Area Telemetered Digital Seismograph Network for providing the seismic data. Authors are also grateful to anonymous referees for constructive suggestions. 


\section{References}

Bensen G D, Ritzwoller M H, Barmin M P, Levshin A L, Lin F, Moschetti M P, Shapiro N M and Yang Y (2007). Processing seismic ambient noise data to obtain reliable broad-band surface wave dispersion measurements. Geophys J Int 169: 1239-1 260.

Campillo M and Paul A (2003). Long-range correlations in the diffuse seismic code. Science 299: 547-549.

Gerstoft P and Tanimoto T (2007). A year of microseisms in southern California. Geophys Res Lett 34: L20304, doi: 10.1029/2007GL031091.

Guo B, Liu Q Y, Chen J H and Li S C (2002). Test of epicenter determination of microearthquakes recorded by the digital seismic network in capital circle. Seismology and Geology 24(3): 453-460 (in Chinese with English abstract).

Larose E, Derode A, Campillo M and Fink M (2004). Imaging from one-bit correlations of wideband diffuse wavefields. $J$ Appl Phys 95: 8 393-8 399.

Roux P, Kuperman W A and the NPAL Group (2004). Extracting coherent wavefronts from acoustic ambient noise in the ocean. J Acoust Soc Am 116: 1 995-2 003

Roux P, Sabra K G, Kuperman W A and Roux A (2005). Ambient noise cross-correlation in free space: Theoretical approach. J Acoust Soc Am 117 79-84.

Ritzwoller M H, Shapiro N M, Pasyanos M E, Bensen G D and Yang Y (2005). Short period surface wave dispersion measurements from ambient seismic noise in North Africa, the Middle East, and Central Asia. Proceedings of the 27th Seismic Research Review: Ground-Based Nuclear Explosion Monitoring. Palm Springs, CA (Sept 20 - 22, 2005).

Sabra K G, Gerstoft P, Roux P, Kuperman, W A and Fehler M C (2005a). Extracting time-domain Greens function estimates from ambient seismic noise. Geophys Res Lett 32: L03310, doi: 10.1029/2004GL021862.

Sabra K G, Gerstoft P, Roux P, Kuperman W A and Fehler M C (2005b). Surface wave tomography from miscroseisms in Southern California. Geophys Res Lett 32: L14311, doi:10.1029/2005GL023155.

Sanchez-Sesma F J, Perez-Ruiz J A, Campillo M and Luzon F (2006). Elastodynamic 2D Green function retrieval from cross-correlation: canonical inclusion problem. Geophys Res Lett 33: L13305, doi:10.1029/2006GL026454.

Shapiro N M and Campillo M (2004). Emergence of broadband Rayleigh waves from correlations of the ambient seismic noise. Geophys Res Lett 31: L07614, doi:10.1029/2004GL019491.

Shapiro N M, Campillo M, Stehly L and Ritzwoller M H (2005). High resolution surface wave tomography from ambient seismic noise. Science 307: $1615-1617$.

Snieder R (2004). Extracting the Green's function from the correlation of coda waves: A derivation based on stationary phase. Phys Rev E 69: 046610 .

Tanimoto T, Ishimaru S and Alvizuri C (2006). Seasonality in particle motion of microseisms. Geophys J Int 166: 253-266.

Wapenaar K (2004). Retrieving the elastodynamic Green's function of an arbitrary inhomogeneous medium by cross correlation. Phys Rev Lett $\mathbf{9 3}$ : 254301.

Wapenaar K, Fokkema J and Snieder R (2005). Retrieving the Green's function in an open system by cross correlation: A comparison of approaches. $J$ Acoust Soc Am 118: 2 783-2 786.

Wapenaar K (2006). Green's function retrieval by cross-correlation in case of one-sided illumination. Geophys Res Lett 33: L19304, doi: 10.1029 /2006GL027747.

Weaver R L and Lobkis O I (2001). Ultrasonics without a source: Thermal fluctuation correlation at MHz frequencies. Phys Rev Lett 87: 134301.

Weaver R L and Lobkis O I (2004). Diffuse fields in open systems and the emergence of the Green's function. J Acoust Soc Am 116: 2 731-2 734 